http://jmscr.igmpublication.org/home/

ISSN (e)-2347-176x ISSN (p) 2455-0450

crossref DOI: https://dx.doi.org/10.18535/jmscr/v7i10.135

Journal Of Medical Science And Clinical Research

IGM Publication

An Official Publication of IGM Publication

\title{
An Unusual Case of Rhabdomyolysis - Pheniramine Maleate over Dose
}

\author{
Authors \\ S. Pravin ${ }^{\text {1* }}$, R. Aswinth ${ }^{2}$, S. Sudharsan ${ }^{3}$ \\ ${ }^{1}$ Post Graduate, ${ }^{2}$ Assistant Professor, ${ }^{3}$ Professor \\ Department of General Medicine, Rajah Muthiah Medical College Hospital, Chidambaram, \\ Tamil Nadu, India \\ *Corresponding Author
}

S. Pravin

\begin{abstract}
Antihistamines are effortlessly to be had over the counter medicines, that are frequently taken in overdoses. The typical effect is observed by means of the anticholinergic outcomes of those medicines. We record a case of a suicide in a male, wherein ingestion of antihistamine pheniramine maleate will cause nontraumatic rhabdomyolysis. Rhabdomyolysis and acute renal failure is a hardly ever said but probably serious problem amongst sufferers who present to the emergency after intentional overdoses making early identification intervention important. We also describe the potential mechanism of muscle injury in antihistamine overdose.
\end{abstract}

Keywords: Antihistamine overdose, pheniramine maleate, rhabdomyolysis.

\section{Introduction}

Antihistaminic, pheniramine maleate, is an alkylamine by-product and without problems to be had over the counter that is popularly used for the remedy of allergic reaction and cold signs. We document a case of rhabdomyolysis not complicated through acute renal failure related to high dose of pheniramine maleate ingestion for suicidal reason.

\section{Case Report}

A 47-year-old male brought to emergency branch with an altered sensorium. His household told he had forty tabs of avil $50 \mathrm{mg}$ (pheniramine maleate $45.3 \mathrm{mg} / \mathrm{tab}$ ). The patient was irritable, agitated, and disoriented. The past medical history was not significant. There was history of alcohol abuse for $8 y$ rs with abstinence from alcohol for 1 month.
Clinical examination showed an axillary temperature of $98.4^{\circ} \mathrm{F}$, blood pressure of $140 / 80 \mathrm{~mm} \mathrm{Hg}$, pulse rate of $110 / \mathrm{min}$, breathing rate of $20 / \mathrm{min}$, and pulse oximetry showed an oxygen saturation of ninety $98 \%$ in room air. Systemic examination confirmed dry mucosa and $3 \mathrm{~mm}$ pupils reacting to light. His cardiac, respiratory and abdominal examinations was normal. Neurological evaluation found out a disoriented guy with ordinary motor findings. There were no signs and symptoms of meningeal irritation. Laboratory facts revealed a ordinary hemogram and blood biochemistry. ECG showed no conduction disturbances but sinus tachycardia. Urine routine showed trace albumin, $\mathrm{RBC}$, and pus cells. Urine toxicology was negative for benzodiazepines and opiates. Patient shifted to the intensive care unit for management. Few hours 
later, his urine became cola colored. It become most effective then his family admitted that he had ate up 40 capsules of Avil 50 (pheniramine maleate $45.3 \mathrm{mg} / \mathrm{tab}) 8$ hours prior to admission. Patient used to take this tablet for symptoms of rhinorhoea. There become no different records that would provide an explanation for his excessive colored urine.

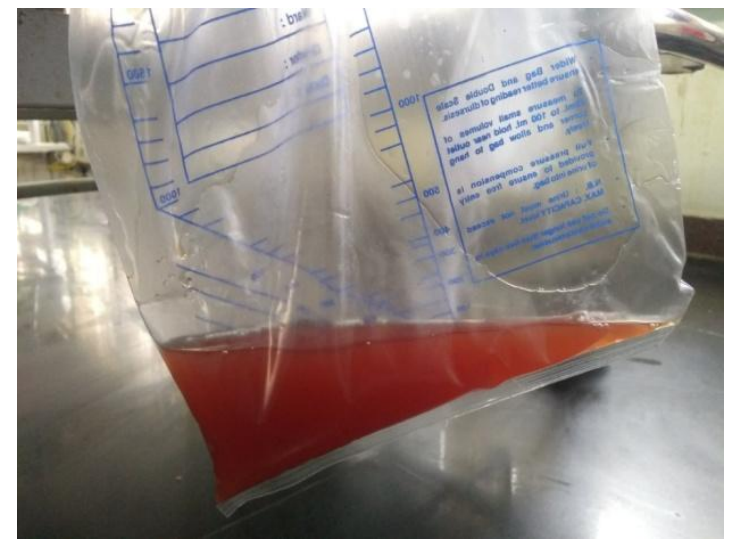

1 day after avil tablet poisoning

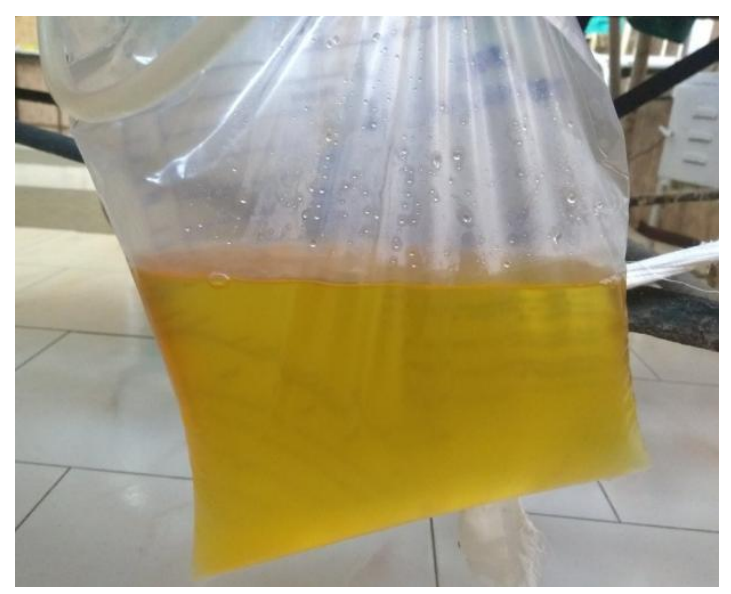

\section{5 days after avil tablet poisoning}

Laboratory statistics on the following day showed total bilirubin $0.8 \mathrm{mg} / \mathrm{dl}$, alkaline phosphatase 204 U/L, ALT 198 IU/L, AST 40 IU/L, and lactate dehydrogenase 412 IU/L. Creatine phosphokinase (CPK) was 10608 IU/L. Urine myoglobin turned into $>30000 \mathrm{ug} / \mathrm{ml}$.

Clinical and laboratory parameters alongside the records have been suggestive of pheniraminecaused rhabdomyolysis. Treatment started out to allow forced alkaline diuresis after which serum creatinine and CPK gradually started lowering, with all odd lab findings normalizing and urine output gradually stepped forward. The affected person was discharged in eight days.

\section{Discussion}

Pheniramine maleate, an alkylamine, belongs to first era of centrally performing $\mathrm{H} 1$ receptor antagonists. Alkylamine derivatives are a few of the maximum effective antihistaminics generating greater CNS stimulation and less drowsiness. They also have aggressive inhibition of muscarinic receptors inflicting anticholinergic aspect consequences. Pheniramine is associated with a enormously high prevalence of seizures $(30 \%)$. It is one of the easily to be had over the counter capsules, and therefore has high capability for misuse. The maximum dose of three $\mathrm{mg} / \mathrm{kg}$ in a day ought to not be used. Our affected person has taken approx. 1812mg. Overdose above the maximum tolerated dose led to appearance of muscarinic features and rhabdomyolysis. Patients who use antihistaminic chronically had tolerance to the psychomotor performance and sedative effects due to autoinduction of hepatic enzymes. ${ }^{[1]}$ Rhabdomyolysis is the rapid breakdown of skeletal muscle and leakage of myocyte contents into extracellular compartment as a result of traumatic (physical) or nontraumatic (chemical or biological) factors. Drugs and alcohol were responsible in eighty one\% cases of nontraumatic rhabdomyolysis. ${ }^{[2]}$ Rhabdomyolysis is normally related to myoglobinuria and might lead to potentially deadly hassle like acute renal failure (ARF), the mechanism of which incorporates obstruction of tubular lumina by means of myoglobin casts, nephrotoxicity due to ferrihemate (breakdown made of myoglobin), and decreased glomerular filteration rate. Among sufferers with nontraumatic rhabdomyolysis, 33\% increase $\mathrm{ARF}$ and $15 \%$ of them require dialysis. ${ }^{[3]}$ Hampel et al. Firstly stated rhabdomyolysis secondary to antihistaminic toxicity leading to renal failure related to myoglobinuria and $\mathrm{CPK}$ fee of 30,000 U/L. ${ }^{[4]}$ There are constrained information in literature concerning rhabdomyolysis because of antihistaminic 
overdose. Only 11 cases of doxylamine and two of diphenhydramine toxicity have been stated to be associated with rhabdomyolysis. ${ }^{[5-7]} \mathrm{CPK}$ stages in these instances ranged from 597 to $78,750 \mathrm{U} / \mathrm{L}$. $\mathrm{ARF}$ requiring hemodialysis came about in most effective one of the sufferers. ${ }^{[8]} \quad$ No case document of rhabdomyolysis with pheniramine has been documented. Our patient had a CPK degree of $10608 \mathrm{U} / \mathrm{L}$ at analysis.

Drug-induced rhabdomyolysis can be because of primary or secondary myotoxic impact. ${ }^{[9]}$ Primary effect is a direct injury at the skeletal myocyte feature and an imbalance among the manufacturing and use of ATP within the muscle fiber. It is hypothesized that drug may exert an immediate toxic impact on sarcolemma, increasing sodium permeability that disrupts calcium homeostasis, leading to alteration in intracellular strength resources and enhancement of intracellular proteolytic enzyme activity. The cell will then go through continuous injury causing rhabdomyolysis. Secondarily, antihistamines by way of impairing the CNS can cause rhabdomyolysis by pressure-precipitated ischemia because of prolonged immobilization. Seizures because of overdose also can contribute to muscle breakdown as formerly mentioned in case series, but there is no seizure or extended immobilization in this patient.

Rhabdomyolysis is most reliably identified by increased levels of creatine phosphokinase within the blood (>5 times). Initial and top CPK levels have a linear relationship with the risk of ARF. Myoglobinuria may additionally precede and clear up prior to an elevation in CPK due to a brief half-life of one to three hours. Therefore, a nil orthotolidine reaction does no longer rule out rhabdomyolysis, and myoglobin is observed in urine most effective in $57 \%$ of patients for the duration of preliminary stage of rhabdomyolysis. ${ }^{[10]}$ Other metabolic abnormalities include metabolic acidosis, elevated lactate dehydrogenase, amino transferases, creatinine, and urea.
Diagnosis of this patient become based totally on clinical, high myoglobinuria and excessive CPK levels. In the absence of trauma, infectious diseases, muscular compression, convulsions, or metabolic disorders, rhabdomyolysis on this affected person was due to pheniramine and was supported via high CPK titers.

Treatment of antihistamine-causing rhabdomyolysis is by recognizing the toxidrome associated with antihistamines, aggressive fluids replacement, careful tracking of electrolytes and renal characteristic, forced alkaline diuresis, and hemodialysis if these conservative measures fail. A postpone in setting up the diagnosis in this situation in all likelihood contributed to the severity of renal failure.

We thought it would be exciting to document this example due to the fact clean availability of antihistamines increases the prevalence of overdose. Hence, relying only on laboratory findings without emphasis on history and clinical presentation may also leave out the diagnosis of antihistaminic poisoning leading to a medico-legal pitfall. More studies could be required to delineate the exact pathophysiology of rhabdomyolysis because of antihistamines apart from the proposed primary and secondary consequences. Antihistamine-brought on rhabdomyolysis is a life-threatening condition, but early recognition and providing aggressive treatment may lead to desirable prognosis, as seen in our patient.

\section{References}

1. Koeppel C, Tenczer J, Ibe K. Poisoning with over the counter doxylamine preparations: an evaluation of 109 cases. Human Toxicol. 1987; 6: 355-9.

2. Prendergast BD, George CF. Druginduced rhabdomyolysis: Mechanisms and management. Postgrad Med J. 1993; 69: 333-6.

3. Gabow PA, Kaehny WD, Kelleher SP. The spectrum of rhabdomyolysis. Med (Baltimore) 1982; 61:141-52. 
4. Hampel G, Horstkotte H, Rumpf KW. Myoglobinuric renal failure due to drug induced rhabdomyolysis. Human Toxicol. 1983; 2:197-201.

5. Frankel D, Dolgin J, Murray BM. Nontraumatic rhabdomyolysis complicating antihistamine overdose. J Clin Toxicol. 1993; 31:493-6.

6. Soto LF, Miller CH, Ognibere AJ. Severe rhabdomyolysis after doxylamine overdose. Postgrad Med. 1993; 93:227-9.

7. Leybishkis B, Fasseas $\mathrm{P}$, Ryan KF. Doxylamine overdose as a potential cause of rhabdomyolysis. Am J Med Sci. 2001; 322:48-9.

8. Emadian SM, Caravati EM, Herr RD. Rhabdomyolysis. A rare adverse effect of diphenhydramine overdose. Am J Emerg Med. 1996; 14: 574-6.

9. Ellenhorn MJ. Ellenhorn's Medical Toxicology, Diagnosis and Treatment of Human Poisoning. 2nd ed. Baltimore: Williams and Wilkins; 1997.

10. Curry SC, Chang D, Connor D. Drug- and toxin-induced rhabdomyolysis. Ann Emerg Med. 1989; 18:1068-84. 